\title{
Vierter Beitrag zur Pilzflora von Tirol ${ }^{1}$ ).
}

Von Fr. Bubák (Tábor in Böhmen) und J. E. Kabát !(Turnau in Böhmen).

Mit Tafel II.

Wie die vorjährige, so basiert auch die vorliegende Abhandlung auf zwei aus Tirol stammenden Pilzkollektionen. Die eine brachte einer von uns (Kabát) von seiner Ferienreise in den Dolomiten, die andere wurde wieder von H. Em. Černý aus Meran zugeschickt. In beiden Sammlungen befinden sich einige sehr interessante Arten, wie aus der folgenden Aufzählung ersichtlich ist.

Tubulina cylindrica (Bull.) DC. An morschen Pinus-Stämmen im Karrerwalde, Eggental.

Albugo Bliti (Biv.) 0. Kuntze. Auf Blättern von Amaranthus retroflexus in Untermais-Meran und bei Pinzolo in Val Rendena. Peronospora effusa (Grev.) Rabh. Auf Chenopodium album bei Birchabruck im Eggentale.

Cronartium asclepiadeum (Willd.) Fries. Auf Vincetoxicum officinale in Val di Génova häufig.

Coleosporium Campanulae (Pers.) Lév. An Blättern von Campanula rotundifolia bei Madonna di Campiglio.

Col. Senecionis (Pers.) Fr. An Blättern von Senecio Fuchsii in der Umgebung des Karrersees im Eggentale.

Pucciniastrum Epilobii (Chaill.) Otth. Uredo an Blättern von Epilobium roseum bei Pinzolo in Val Rendena.

Calyptospora Goeppertiana Kühn. Auf Vaccinium Vitis idaea bei Birchabruck, Wälschnofen und Karrersee im Eggentale.

Uredinopsis filicina (Niessl) Magnus. Auf Phegopteris polypodioides im Val Nambino und bei Madonna di Campiglio.

Gymnosporangium clavariaeforme (Jacq.) Rees. Obermais bei Meran auf Blättern von Crataegus monogyna.

Uromyces Fabae (Pers.) De Bary. An Blättern und Stengeln von Vicia cracca unterhalb Birchabruck im Eggentale.

Ur. Silenes (Schlecht.) Fuckel. Auf Blättern und Stengeln von Silene nutans bei Carisolo in Val Rendena mit Darluca filum (Biv. Beruh.) und Septoria dimera Sace.

Puccinia Arenariae (Schum.) Wint. Auf Stellaria nemorum bei Karrersee im Eggentale.

Pucc. Veronicarum DC. Auf Blättern von Veronica urticaefolia Jaeq. in Val di Génova.

Pucc. Glechomatis DC. An Blättern von Glechoma hederaceum in $\nabla$ al di Génova.

Pucc. Salviae Unger. Auf Salvia glutinosa unterhalb Birchabruck im Eggentale und im $V$ al di Génova.

Pucc. conglomerata (Strau@) Schmidt et Kunze. Auf Blättern von Homogyne alpina im Karrerwalde, Eggental.

$\left.{ }^{1}\right)$ Siehe diese Zeitschrift 1899 Nr. 4, 1900 Nr. 8, 1904 Nr. 4, 5.

Osterr. botan. Zeitschrift. 2. Heft. 1905.

6 
Pucc. Morthieri Körn. Auf Blättern von Geronium silvaticum bei Madonna di Campiglio.

Pucc. chondrillina Bubák et Sydow. Auf Chondrilla juncea bei Meran.

Pucc. Taraxaci (Rbh.) Plowr. An Blättern von Taraxacum officinale bei Pinzolo in. $\nabla$ al Rendena.

Pucc. Centaureae Mart. An Blättern von Centaurea Jacea bei Carisolo in Val Rendena.

Pucc. Rumicis scutati (DC.) Wint. Auf Rumex scutatus bei Pinzolo, Carisolo in Val Rendena und in Val Nambino.

Pucc. Cirsii lanceolati Schröt. An Blättern von Cirsium lanceolatum in Val di Génova.

Pucc. Convolvuli (Pers.) Cast. Auf Convolvulus sepium in Untermais bei Meran, Aecidien am 21. Juli, II + III am 6. August.

Pucc. Menthae Pers. An Blättern von Mentha silvestris bei Pinzolo in $\mathrm{Val}$ Rendena.

Pucc. Mougeotii Lagerh. An Blättern und Stengeln von Thesium alpinum auf den Bewallerwiesen im Eggentale (Uredo., Teleut., 25. VII.).

Pucc. Pimpinellae (Strauß.) Link. Auf Pimpinella magna bei Pinzolo und Carisolo in $\mathrm{Val}$ Rendena.

Pucc. Violae (Schum.) DC. An den Blättern von Viola silvestris in Val di Génova.

Pucc. graminis Pers. Auf Triticum repens bei Meran.

Pucc. Phragmitis (Schum.) Körn. Auf Phragmites communis bei Meran.

Pucc. Polygoni Pers. An Blättern von Polygonum convolvulus bei Pinzolo in Val Rendena.

Pucc. Polygoni vivipari Karst. An Blättern von Polygonum vivi parum bei Madonna di Campiglio. Hierher gehört auch der Pilz von Pedracses in Val Badia, welcher im I. Beitrage (diese Zeitschr. 1899, Nr. 4) als Pucc. Bistortae DC. (kollektiv) veröffentlicht wurde.

Phragmidium Potentillae (Pers.) Karst. Auf Potentilla argentea Untermais bei Meran.

Phr. Rosae alpinae (DC.) Wint. Auf Rosa alpina im Eggentale häufig.

Phr. Rubi (Pers.). An Blättern von Rubus caesius unterhalb Birchabruck im Eggentale.

Uredo alpestris Schröt. An Blättern von Viola biflora bei Madonna di Campiglio.

Exobasidium Rhododendri Cramer. An Rhododendron ferrugineum in der Umgebung von Madonna di Campiglio häufig.

Peniophora cinerea (Fr.) Cooke, Schlo@ Pienzenen bei Meran auf Rosa and Alnus glutinosa.

Corticium obscurum Pers. Auf dürren Ästen von Castanea vesca in Meran. 
Sphaerotheca Castagnei Lév. An Blättern von Humulus lupulus bei Birchabruck im Eggentale und bei Pinzolo in Val Rendena. Erysiphe Polygoni DC. Meran auf Polygonum aviculare und Convolvulus arvensis.

Er. Cichoriacearum DC. Auf Senecio Fuchsii bei Karrersee im Eggentale.

Microsphaera Guarinonii Briosi et Cav. An Blättern von Cytisus Laburnum in Gesellschaft mit Septoria Cytisi Desm. in Val di Génova. Der Pilz war bisher nur aus Italien bekannt.

Micr. Astragali (DC.) Trev Auf Blättern und Blattstielen von Astragalus glycyphyllus in Val di Génova.

Lasiobotrys Lonicerae Kunze. Auf Blättern von Lonicera coerulea in der Umgebung von Karrersee im Eggentale.

Pleonectria Lamyi Desm. Obermais bei Meran auf Berberis vulgaris.

Cucurbitaria Coronillae (Fr.) Sace. Au trockenen Zweigen von Coronilla Emerus unterhalb Birchabruck im Eggentale.

Didymosphaeria nobilis Sace. Auf trockenen Ästen von Laurus nobilis Meran.

Didymella Cadubriae Sace. Auf trockenen Ästen von Berberis vulgaris in Obermais bei Meran.

Didymella Castaneae (Togn.) Bubák. (Sphaerella Castaneae Tognini in Contr. Mic. Tose. pg. 8).

Perithecien endlich oberflächlich über die Äste zerstreut, linsenförmig, 200-250 $\mu$ im Durchmesser, 90-100 $\mu$ hoch, kohlig, schwarz, oft schwach glänzend, endlich mit breiter, zentraler, rundlicher Mündung, von dunkelbraunem, parenchymatischem Gewebe; Asci zylindrisch oder verkehrt keulenförmig, unten plötzlich in einen kurzen Stiel zusammengezogen, oben abgerundet, 33-55 $\mu$ lang, $11-15 \mu$ breit, 8sporig, umgeben von ebenso langen, gekrümmten, knorrigen oder mit kurzen Ästchen versehenen, $2-2 \cdot 5 \mu$ breiten Parafysen; Sporen eiförmig, zweizellig, die untere Zelle kürzer und breiter als die obere, beide abgerundet, $13-17 \mu$ lang, $4 \cdot 5-6 \cdot 5 \mu$ breit, hyalin.

Meran, auf alten Ästen von Castanea vesca, leg. im September 1904 Em. Černý.

Der vorliegende Pilz paßt gut auf die kurze Diagnose Tognini's, wie sie in Saccardo, Sylloge XI., pg. 298, wiedergegeben ist.

Leptosphaeria derasa (Berk. et Br.) Thüm. Auf alten Stengeln von Senecio Fuchsii im Karrerwalde. Eggental.

Lept. ogilviensis (Berk. et Br.) Ces. et Not. Auf trockenen Stengeln von Solidago virgaurea zwischen Birchabruck und Eggen im Eggentale.

Lept. Senevionis (Fuckel) Wint. An trockenen Stengeln von Senecio Fuchsii im Karrerwalde, Eggental. 
Lept. vagabunda Sace. Auf absterbenden Ästen von Gleditschia triacanthos in Meran.

Mamiania Coryli (Butsch) Ces. et Not. An Blättern von Corylus Avellana in Val di Génova.

Valsa horrida Nitschke. Untermais bei Meran auf abgestorbenen Ästen von Betula alba.

Anthostoma alpigenum (Fuckel) Sacc. An trockenen Zweigen von Lonicera alpigena bei Karrersee im Eggentale.

Diatrypella aspera (Fries) Nitschke. Untermais bei Meran auf toten Ästen von Alnus glutinosa.

Diatr. verruciformis (Ehrh.) Nitschke. Auf abgestorbenen Ästen von Salix in Untermais bei Meran.

Hypoxylon fuscum (Pers.) Fries. Auf toten Ästen von Alnus glutinosa in Meran.

Dothidella betulina (Fries) Sace. Auf lebenden Blättern von Betula verrucosa in Val di Génova.

Dothidea Sambuci (Pers.) Fries forma moricola Sace. Aut toten Ästen von Morus alba in Meran und bei Pinzolo in Val Rendena.

Plowrightia Berberidis (Wahlb.) Sacc. An trockenen Zweigen von Berberis vulgaris bei Birchabruck und Wälschnofen im Eggentale.

Plowr. ribesia (Pers.) Sacc. Auf toten Ästen von Ribes rubrum in Meran.

Phyllachora Ulmi (Duv.) Fuckel. Auf løbenden Blättern von Ulmus campestris in Meran.

Hysterographium Fraxini (Pers.) De Not. Auf trockenen Ästen von Fraxinus excelsior in Meran.

Hypoderma virgultorum DC. f. Vincetoxici Duby. An trockenen Stengeln von Vincetoxicum officinale in Val di Génova.

Sphaeropezia Vaccinii Rehm. An trockenen Blättern von Vaccinium Vitis idaea im Karrerwalde, Eggental, häufig.

Rhytisma salicinum (Pers.) Fries. Auf Blättern von Salix glabra bei Birchabruck im Eggentale.

Tapesia Rosae (Pers.) Rehm. An faulenden Zweigen vou Rosa alpina bei Karrersee im Karrerwalde und auf den angrenzenden Wiesen, Eggental.

Pirottaea gallica Sace. An faulenden Stengeln von Senecio Fuchsii im Karrerwalde, Eggental.

Phyllosticta camelliaecola Brun., var. n. meranensis Bubák. Meran auf lebenden Blättern von Camellia japonica (12. April 1904, leg. E. Černý). Der vorliegende Pilz stimmt habituell (nach der Diagnose) mit der Brunaud'schen Spezies überein, nur die Sporen sind etwas abweichend, nämlich zylindrisch, $3-5 \mu$ lang, $1-1 \cdot 5 \mu$ breit.

Da mir aber nur sehr wenig Material vorliegt, so stelle ich den Pilz, welcher vielleicht eine neue Spezies darstellt, vorläufig nur als Varietät auf. Vom Typus weicht sie durch bakterienförmige, kleinere Sporen ab. 
Phyllosticta latemarensis Kabát et Bubák n. sp. Flecken beiderseits, klein, unregelmässig länglich, schwarz, zusammenfliessend und bald größere Partien oder das ganze Blatt schwarz verfärbend.

Fruchtgehäuse beiderseits, zerstreut, seltener zu mehreren aneinander gedrängt oder herdenweise, schwarz, kuglig, 60 bis $160 \mu$ im Durchmesser, eingewachsen, dauernd bedeckt, endlich die Epidermis sprengend und mit rundem Porus sich öffnend, von festem, ziemlich dichtem, fast schwarzem, parenchymatischem Gewebe, mit ziemlich dichten, wurmförmigen, dunkelbraunen Hyphen im Mesophyll.

Sporen massenhaft, stäbchenförmig, an den Enden abgerundet, gerade oder etwas gebogen, $4-6 \mu$ lang, $0 \cdot 75-1 \mu$ breit, hyalin.

Sporenträger zylindrisch, gegen die Spitze verjüngt, etwa $10 \mu$ lang, $2 \mu$ breit, hyalin.

An absterbenden Blättern von Colchicum autumnale auf Bergwiesen am Costalungapa (5 $(1700 \mathrm{~m})$ unter dem Latemargebirge im Eggentale, in Gesellschaft von Septoria gallica Sacc. et Syd. am 24. Juli 1904, legit Kabát.

Phyllosticta lupulina Kabát et Bubák n. sp. Flecken beiderseits deutlich sichtbar, über die ganze Blattfläche mehr oder weniger dicht zerstreut, klein, höchstens $5 \mathrm{~mm}$ breit, eckig oder unregelmäßig rundlicheckig, oft zusammenfließend, trocken, beiderseits eingesunken, weißgrau, mit schmaler, purpurbrauner, oft fehlender Umrandung.

Fruchtgehäuse oberseits, zerstreut, punktförmig, schwarz, kuglig, schwach abgeflacht, trocken einsinkend, eingewachsen, später die Epidermis mit dem Scheitel zerreißend, 50-70 $\mu$ im Durchmesser, oben aus dunkelbraunem oder rußfarbigem, im übrigen Teile aus hellbraunem, ziemlich derbem, dichtem, parenchymatischem Gewebe.

Sporen sehr zahlreich, eiförmig, ellipsoidisch oder länglich, an den Enden abgerundet, $3-7 \mu$ lang, $2-3 \mu$ breit, hyalin auf kurzen, hyalinen Sporenträgern.

Auf noch lebenden und absterbenden Blättern von Humulus lupulus zwischen Birchabruck und Unter-Eggen im Eggentale. gemeinschaftlich mit Septoria Humuli West. am 16. Juli 1904, leg. Kabát.

Von Phyllosticta Humuli Sacc. et Speg. durch kleinere, mündungslose Pykniden und kleinere Conidien verschieden.

Phyllosticta iliciseda Sace. in Atti Congr. bot. di Palermo 1902. Auf lebenden Blättern von Quercus Ilex in Meran.

Die Bestimmung dieses schönen Pilzes verdanke ich Herrn Prof. P. A. Saccardo. 
Phyllosticta Trollii Trail. Auf Blättern von Trollius europaeus in Gesellschaft mit Ramularia Trollii (Jacz.) im Val Mortitz unter der Sellagruppe (25. Juli 1901).

Phoma cinerascens Sacc. In Meran auf toten Ästen von Ficus carica (Mai 1904).

Phomopsis Lactucae (Sace.) Bubák. Auf trockenen Stengeln und Ästen von Lactuca sativa in Untermais bei Meran (September 1904, leg. E. Cerný).

Der tirolische Pilz bedeckt die trockenen Stengel und Äste in weitläufigen Herden mit schwarzen Pykniden. Dieselben sind eingewachsen, 200-450 $\mu$ breit, im Umrisse rundlich oder länglich, auf 150-200 $\mu$ abgeflacht. Ihr Gewebe ist parenchymatisch, unten und au den Seiten hellkastanienbraun, oben fast schwarz und bildet daselbst $100-125 \mu$ hohen, $80-90 \mu$ breiten, zylindrischen oder konischen Schnabel, mittelst welchem die Epidermis durchgebrochen wird. Am Rande der Schnabelöffnung befinden sich in kreisförmiger Anordnung, dunkelbraune, fransenartige Hyphen, welche aus den Randzellen hervorwachsen.

In den Pykniden befinden sich zweierlei Konidien. Die phomaartigen sind elliptisch oder zylindrisch-spindelförmig, beiderseits abgerundet oder unten zugespitzt, $7-15 \mu$ lang, $2-3 \mu$ breit, hyalin, mit 2 Öltropfen; ihre Sporenträger sind schmal flaschenförmig, 10-20 $\mu$ lang, unten strauchartig verbunden, etwa so breit wie die Sporen.

Außer diesen, sozusagen, normalen Sporen, sind ebenfalls zahlreich auch septoriaartige Konidien vorhanden. Dieselben sind am oberen Ende hackenförmig gebogen, 15-25 $\mu$ lang, $1 \mu$ breit, hyalin, einzellig, mit undeutlichen Öltropfen; sie stehen auf kurzen ziemlich dicken konischen Trägern, von welchen sie sich sehr leicht abtrennen.

Sac card $0^{1}$ ) hält diese letzten Konidien für Konidienträger. Da sie sich aber äuferst leicht und noch in der Pyknide von den eigentlichen Träger abtrennen, so bin ich geneigt dieselben als Sporen aufzufassen.

Was die Benennung des Pilzes betrifft, so ist es wirklich, wie schon Saccardo l. c. betont, besser und ratsamer, denselben und alle ähnlichen Phoma-Arten, die nach demselben Mykologen Konidienstadien zu Diaporthe-Arten vorstellen, von Phoma abzutrennen und in der Gattung $\mathrm{Phomopsis}$ zusammenzufassen.

Phoma diversispora Bubák n. sp.

Pykniden herdenweise, ohne Fleckenbildung, linsenförmig, von der Epidermis bedeckt und dieselbe mit kleiner, schwarzer, deutlich unter der Lupe sichtbarer Papille durchstechend, braun

1) Saccardo, Sylloge fungorum III, pg. 66. 


\section{$2 \mathrm{BHL}$ Biodiversity Heritage Library}

Bubák, František and Kabát, J E . 1905. "Vierter Beitrag zur Pilzflora von Tirol." Oesterreichische botanische Zeitschrift 55, 73-79.

View This Item Online: https://www.biodiversitylibrary.org/item/36435

Permalink: https://www.biodiversitylibrary.org/partpdf/246372

\section{Holding Institution}

University Library, University of Illinois Urbana Champaign

\section{Sponsored by}

University of Illinois Urbana-Champaign

\section{Copyright \& Reuse}

Copyright Status: Public domain. The BHL considers that this work is no longer under copyright protection.

This document was created from content at the Biodiversity Heritage Library, the world's largest open access digital library for biodiversity literature and archives. Visit BHL at https://www.biodiversitylibrary.org. 